\title{
ON THE ZEROS OF LINEAR RECURRENCE SEQUENCES
}

\author{
FRANCESCO AMOROSO AND EVELINA VIADA \\ Laboratoire de mathématiques Nicolas Oresme, CNRS UMR 6139 \\ Université de Caen, Campus II, BP 5186 \\ 14032 Caen Cedex, France \\ Department of Mathematics \\ University of Basel, Rheinsprung 21 \\ 4051 Basel, Suisse \\ Supported by the Fonds National Suisse (FNS)
}

\begin{abstract}
We improve by one exponential W. M. Schmidt's estimate for the Skolem-Mahler-Lech theorem on the number of arithmetic progressions describing the zeros of a linear recurrence sequence.
\end{abstract}

\section{INTRODUCTION}

A linear recurrence sequence of order $t \geq 1$ is a sequence $\left\{u_{m}\right\}_{m \in \mathbb{Z}}$ of elements in an algebraically closed field $K$ of characteristic zero which satisfies a minimal relation

$$
u_{m+t}=c_{1} u_{m+t-1}+\cdots+c_{t} u_{m} \quad(m \in \mathbb{Z})
$$

with $c_{1}, \ldots, c_{t} \in K$. We say that $\left\{u_{m}\right\}$ is simple if its companion polynomial $\mathcal{P}(z)=z^{t}-c_{1} z^{t-1}-\cdots-c_{t}$ has only simple roots. Let

$$
\mathcal{S}\left(u_{m}\right)=\left\{k: u_{k}=0\right\} .
$$

The Skolem-Mahler-Lech theorem asserts that for an arbitrary linear recurrence sequence $\left\{u_{m}\right\}$ of order $t \geq 1$ the set $\mathcal{S}\left(u_{m}\right)$ is a finite union of arithmetic progressions, where we adopt the following convention: single elements of $\mathbb{Z}$ are trivial arithmetic progressions. In [4], J.-H. Evertse, H.P. Schlickewei and W. Schmidt prove that for a simple linear recurrence sequence of order $t$ the set $\mathcal{S}\left(u_{m}\right)$ is the union of at most

$$
\exp \left\{(6 t)^{3 t}\right\}
$$

arithmetic progressions.

Let $K$ be a field of characteristic 0 . Let $\left(a_{1}, \ldots, a_{n}\right) \in\left(K^{*}\right)^{n}$ and $\Gamma$ be a subgroup of $\left(K^{*}\right)^{n}$ of finite rank $r$. Let us consider the equation

$$
a_{1} \alpha_{1}+a_{2} \alpha_{2}+\ldots+a_{n} \alpha_{n}=1 \quad \text { with } \quad \boldsymbol{\alpha} \in \Gamma,
$$

We say that a solution of (1.1) is non-degenerate if no subsum of the left hand side of (1.1) vanishes. The result on linear recurrence sequences of [4], is a quite straightforward corollary of their bound for the number of nondegenerate solutions of the equation (1.1). In turns, this last estimate depends on two different tools. An application of the Subspace Theorem gives an estimate on the "large" solutions of equation (1.1). To handle the "small" 
solutions usually one applies a gap principle. For this purpose one needs a lower bound for the height of a small solution. In the quoted paper they use a result of Schmidt [5]. In [1], corollary 1.4, we considerably improved such a bound. Thus we can save one exponential in the bound of [4] for the number of non-degenerate solutions of the equation (1.1) (see ([1], theorem 6.2). As a further application, we obtained ([1], corollary 6.3) that, for a simple linear recurrence sequence of order $t$, the set $\mathcal{S}\left(u_{m}\right)$ is the union of at most

$$
(8 t)^{4 t^{5}}
$$

arithmetic progressions.

In [7], Schmidt generalized the zero estimate of [4] to arbitrary linear recurrence sequence of order $t$. He proved that $\mathcal{S}\left(u_{m}\right)$ is the union of at most

$$
\exp \exp \exp (20 t)
$$

arithmetic progressions. This was recently improved to

$$
\exp \exp \exp (\sqrt{11 t} \log t)
$$

by Allen (see [3]). The key change in his proof is an improvement on [7], lemma 2 on linear independence.

One of the fundamental tools in Schmidt's proof is the estimate of [4] for the number of non-degenerate solutions of the equation (1.1). The aim of the present paper is to briefly show how our results in [1] allow us to substantially improve (1.2), saving an exponential.

Theorem 1.1. Let $\left\{u_{m}\right\}$ be a linear recurrence of order $t$. Then the set $\mathcal{S}\left(u_{m}\right)=\left\{k: u_{k}=0\right\}$ is the union of at most

$$
\exp \exp (70 t)
$$

arithmetic progressions.

We then improve some other bounds. Let $\alpha_{1}, \ldots, \alpha_{k}$ be the distinct roots of the companion polynomial $\mathcal{P}$ and let $a$ be the maximum of their multiplicity in $\mathcal{P}$. In [7], Schmidt also proves that $\mathcal{S}\left(u_{m}\right)$ is a union of at most

$$
\exp \exp \left(30 a k^{a} \log k\right)
$$

arithmetic progressions. Theorem 1.1 suggests that one could possibly improve by one exponential also this last estimate. Unfortunately, we are not able to do that. This is due to the double exponential growth in $t$ of the function $Z(t, T)$ which bounds in equation (3.1) the number of such arithmetic progressions. However, we can successfully treat the case of non-degenerate sequence. We recall that a sequence is non-degenerate if no quotient $\alpha_{i} / \alpha_{j}$ $(1 \leq i<j \leq k)$ is a root of unity. For non-degenerate sequence the SkolemMahler-Lech simply asserts that $\mathcal{S}\left(\left\{u_{m}\right\}\right)$ is a finite set. Its cardinality is called the zero multiplicity of the sequence $\left\{u_{m}\right\}$. The following result improves by one exponential the main theorem of $[6]$.

Theorem 1.2. Let $\left\{u_{m}\right\}$ be a non-degerate linear sequence whose companion polynomial has $k$ distinct roots with multiplicity $\leq a$. Then, the zero multiplicity of $\left\{u_{m}\right\}$ is bounded by

$$
\left(8 k^{a}\right)^{8 k^{6 a}} \leq \exp \left(32 a k^{6 a} \log k\right) .
$$


We finally remark that in theorem 1.1, one could naturally think to combine our improvement with Allen's refined version of [7], lemma 2, to obtain a lower bound of the shape

$$
\exp \exp (c \sqrt{t} \log t) .
$$

We can not do this, because of the double exponential growth in $t$ of the function $Z(t, T)$ and because of the double exponential growth in $n$ for the number of systems of 3-elements sets (see page 9 for some more details).

Acknowledgements. We thank Y. Bugeaud for calling our attention on Schmidt's result [7].

\section{Ingredients of the Proof}

2.1. Auxiliary results. In what follows we sum up an improved version of some lemmas of [6] which we need in order to obtain the final result. Essentially, we replace the main results of [4] by those of [1], section 6 . Let us sketch the necessary computations.

For integers $q \geq 1$ and $r \geq 0$ we define

$$
C(q, r)=(8 q)^{4(q-1)^{4}(q+r)} \text {. }
$$

Lemma 2.1 (Counterpart to [6], lemma 4). Let $\Gamma$ be a finitely generated subgroup of $\left(\mathbb{C}^{*}\right)^{q}$ of rank $r$, and let $a_{1}, \ldots, a_{q} \in \mathbb{C}^{*}$. Then, up to a factor of proportionality, the equation

$$
a_{1} x_{1}+\cdots+a_{q} x_{q}=0
$$

has at less than $C(q, r)$ non-degenerate solutions $\mathbf{x} \in \Gamma$.

Proof. This is an inhomogeneous version of theorem 6.2 of [1]. Indeed, set $n=q-1, b_{i}=-a_{i} / a_{q}$ and $y_{i}=x_{i} / x_{q}(i=1, \ldots, n)$. Then, the equation becomes

$$
b_{1} y_{1}+\cdots+b_{n} y_{n}=1
$$

with $\mathbf{y}=\left(y_{1}, \cdots, y_{n}\right)$ in a subgroup of rank $\leq r$. By theorem 6.2 of [1] this last equation has at most

$$
(8 n)^{4 n^{4}(n+r+1)}<C(q, r)
$$

non-degenerate solutions.

For $\boldsymbol{\alpha} \in \mathbb{P}^{n}(\overline{\mathbb{Q}})$ we denote by $h(\boldsymbol{\alpha})$ the absolute, logarithmic Weil height of $\boldsymbol{\alpha}$. For $\left.\boldsymbol{\alpha}=\left(\alpha_{1}, \cdots, \alpha_{n}\right)\right) \in\left(\overline{\mathbb{Q}}^{*}\right)^{n}$ we let $\hat{h}(\boldsymbol{\alpha})=h\left(\left(1: \alpha_{1}: \cdots: \alpha_{n}\right)\right)$.

Lemma 2.2 (Counterpart to [6], lemma 5). Let $q>1$ and let $\Gamma$ be a finitely generated subgroup of $\left(\overline{\mathbb{Q}}^{*}\right)^{q}$ of rank $r$. Consider the set $S$ of solutions of the equation

$$
z_{1}+\cdots+z_{q}=0
$$

with $\mathbf{z}=\mathbf{x y}, \mathbf{x} \in \Gamma, \mathbf{y} \in \mathbb{Q}^{q}$ and

$$
h(\mathbf{y}) \leq \frac{1}{4 q^{2}} h(\mathbf{x}) .
$$


Then $S$ is contained in the union of less than $C(q, r)$ proper linear subspaces of the $(q-1)$-dimensional linear space defined by (2.1).

Proof. Set $n=q-1$. As in the proof of [6], it is enough to prove the following inhomogeneous version of this lemma. Let $\Gamma$ be a finitely generated subgroup of $\left(\overline{\mathbb{Q}}^{*}\right)^{n}$ of rank $r$. Let $S^{\prime}$ be the set of solutions of the equation $z_{1}+\cdots+z_{n}=1$ with $\mathbf{z}=\mathbf{x y}, \mathbf{x} \in \Gamma, \mathbf{y} \in\left(\mathbb{Q}^{*}\right)^{n}$ and $\hat{h}(\mathbf{y}) \leq \frac{1}{4 n^{2}} h(\mathbf{x})$. Then $S^{\prime}$ is contained in the union of not more than $C(q, r)$ proper linear subspaces of $\left(\overline{\mathbb{Q}}^{*}\right)^{n}$. We follow the proof of [6] replacing Theorem 2.1 of [4] by Theorem 6.1 of [1]. Then $S^{\prime}$ is contained in the union of not more than

$$
n+2^{30 n^{2}}\left(21 n^{2}\right)^{r}+(8 n)^{\left(6 n^{3}\right)(n+r)}
$$

proper linear subspaces of $\left(\overline{\mathbb{Q}}^{*}\right)^{n}$. We clearly assume $n \geq 2$. Using $2=$ $16^{1 / 4} \leq(8 n)^{1 / 4}$ and $1+7.5 x^{2}+6 x^{4} \leq 4 x^{4}(x+1)$ for $x \geq 2$, we see that

$$
\begin{aligned}
n+2^{30 n^{2}}\left(21 n^{2}\right)^{r}+(8 n)^{\left(6 n^{3}\right)(n+r)} & \leq(8 n)^{1+7.5 n^{2}+2 r+\left(6 n^{3}\right)(n+r)} \\
& \leq(8 n)^{4 n^{4}(n+r+1)}<C(q, r) .
\end{aligned}
$$

For a non-zero polynomial $P \in \mathbb{C}[X]$ we put $t(P)=1+\operatorname{deg}(P)$ and we agree that $t(0)=0$. For a vector $\mathbf{P}=\left(P_{1}, \ldots, P_{k}\right) \in(\mathbb{C}[X])^{k}$, define $t(\mathbf{P})=t\left(P_{1}\right)+\cdots+t\left(P_{k}\right)$ and $a(\mathbf{P})=\max _{i} t\left(P_{i}\right)$.

Let $\alpha_{1}, \ldots, \alpha_{k} \in\left(\overline{\mathbb{Q}}^{*}\right)^{k}$ be algebraic numbers and let $P_{1}, \ldots, P_{k} \in \overline{\mathbb{Q}}[X]$ be non-zero polynomials. We consider the polynomial-exponential equation

$$
P_{1}(x) \alpha_{1}^{x}+\cdots+P_{k}(x) \alpha_{k}^{x}=0 .
$$

Put for simplicity $t=t(\mathbf{P})$ and $t^{*}=1+a(\mathbf{P})$. Assume $t \geq 3$ and $\hbar \in(0,1]$. We suppose that

Let us define

$$
\max _{i, j} h\left(\left(\alpha_{i}: \alpha_{j}\right)\right) \geq \hbar .
$$

$$
E=16 t^{2} \cdot t^{*} / \hbar, \quad F=(8 t)^{4(t-1)^{4}(t+2)}+5 E \log E .
$$

Lemma 2.3 (Counterpart to [6], lemma 7). There exist $k$-tuples $\mathbf{P}^{(w)}=$ $\left(P_{1}^{(w)}, \ldots, P_{k}^{(w)}\right) \neq(0, \ldots, 0)(1 \leq w<F)$ of polynomials with

$$
\begin{array}{ll}
\operatorname{deg} P_{i}^{(w)} \leq \operatorname{deg} P_{i} & (1 \leq w<F, 1 \leq i<k) \\
\operatorname{deg} P_{k}^{(w)}<\operatorname{deg} P_{k} & (1 \leq w<F)
\end{array}
$$

such that every solution $x \in \mathbb{Z}$ of (2.2) satisfies

$$
P_{1}^{(w)}(x) \alpha_{1}^{x}+\cdots+P_{k}^{(w)}(x) \alpha_{k}^{x}=0 .
$$

for some $w \in[1, F)$.

Proof. We replace in Schmidt's proof lemma 5 of [6] by lemma 2.2. From (4.10) of [6] and from the discussion below that formula, we see that we can take

$$
F=C(t, 2)+5 E \log E=(8 t)^{4(t-1)^{4}(t+2)}+5 E \log E .
$$


Let $\alpha, \beta$ be complex numbers. We write $\alpha \approx \beta$ if $\alpha, \beta$ are non-zero and $\alpha / \beta$ is a root of unity.

Let $a_{1}, \ldots, a_{q}, \alpha_{1}, \ldots, \alpha_{q} \in \mathbb{C}$ and

$$
f(x)=a_{1} \alpha_{1}^{x}+\cdots+a_{q} \alpha_{q}^{x} .
$$

Consider a partition of the summands such that $a_{i} \alpha_{i}^{x}$ and $a_{j} \alpha_{j}^{x}$ are in the same sub-part if and only if $\alpha_{i} \approx \alpha_{j}$. After relabeling, one can write

$$
f(x)=f_{1}(x)+\cdots+f_{g}(x)
$$

where

$$
f_{i}(x)=a_{i, 1} \alpha_{i, 1}^{x}+\cdots+a_{i, q_{i}} \alpha_{i, q_{i}}^{x} \quad(i=1, \ldots, g)
$$

with $q_{1}+\cdots+q_{g}=q$ and

$$
\begin{aligned}
& \alpha_{i, j} \approx \alpha_{i, k} \quad \text { when } \quad 1 \leq i \leq g, 1 \leq j, k \leq q_{i} ; \\
& \alpha_{i, j} \not \approx \alpha_{i^{\prime}, k} \text { when } 1 \leq i \neq i^{\prime} \leq g, 1 \leq j \leq q_{i}, 1 \leq k \leq q_{i^{\prime}} \text {. }
\end{aligned}
$$

Lemma 2.4 (Counterpart to [6], lemma 8). All but at most

$$
G(q)=(8 q)^{4(q-1)^{3} q^{2}}
$$

solutions $x \in \mathbb{Z}$ of $f(x)=0$ have $f_{1}(x)=\cdots=f_{g}(x)=0$.

Proof. In the proof of [6], lemma 8, we replace [6] lemma 4 by our lemma 2.1 . From the last formula of [6], p. 258, we see that it is enough to show that $C(q, 1)+2^{q} G(q-1) \leq G(q)$ for $q \geq 2$. This arises from

$$
\begin{aligned}
C(q, 1)+2^{q} G(q-1) & \leq(8 q)^{4(q-1)^{4}(q+1)}+2^{q}(8(q-1))^{4(q-2)^{3}(q-1)^{2}} \\
& \leq\left(1+2^{q}\right)(8 q)^{4(q-1)^{2} \max \left((q-1)^{2}(q+1),(q-2)^{3}\right)} \\
& \leq(8 q)^{q+4(q-1)^{2} \max \left((q-1)^{2}(q+1),(q-2)^{3}\right)} \leq(8 q)^{4(q-1)^{3} q^{2}}
\end{aligned}
$$

(use $x+4(x-1)^{2} \max \left((x-1)^{2}(x+1),(x-2)^{3}\right) \leq 4(x-1)^{3} x^{2}$ for $\left.x \geq 2\right)$.

2.2. Main Proposition. We improve the main Proposition at the beginning of section 3 of [7] replacing in (3.4) of that proposition the value of $H(T)$ by $(8 T)^{4(T-1) T^{4}}$. For the convenience of the reader we recall the statement of this Proposition, which is the core of [7]. Let

$$
M_{j}(\mathbf{X})=a_{1, j} X_{1}+\cdots+a_{k, j} X_{k} \quad(j=1, \ldots n)
$$

be linear forms with algebraic coefficients which are linearly independent over $\mathbb{Q}$. We write $\mathbf{a}_{\mathbf{i}}=\left(a_{i, 1}, \ldots, a_{i, n}\right)$ and assume that each $\mathbf{a}_{\mathbf{i}} \neq(0, \ldots, 0)$ $(i=1, \ldots, k)$. We define $t_{i}$ to be the integer such that

$$
\mathbf{a}_{\mathbf{i}}=\left(a_{i, 1}, \ldots, a_{i, t_{i}}, 0, \ldots, 0\right)
$$

with $a_{i, t_{i}} \neq 0$. Set $t=t_{1}+\ldots+t_{k}$,

$$
\begin{aligned}
& T=\min \left(k^{n}, e^{12 t}\right), \\
& \hbar=\hbar(T)=e^{-6 T^{4}} .
\end{aligned}
$$


Proposition 2.5. Suppose that $\alpha_{1}, \ldots, \alpha_{k}$ are non-zero algebraic numbers. Consider $x \in \mathbb{Z}$ for which

$$
M_{1}\left(\alpha_{1}^{x}, \ldots, \alpha_{k}^{x}\right), \ldots, M_{n}\left(\alpha_{1}^{x}, \ldots, \alpha_{k}^{x}\right)
$$

are linearly independent over $\mathbb{Q}$. These numbers falls into at most

$$
H(T)=(8 T)^{4(T-1) T^{4}}
$$

classes with the following properties. For each class $C$ there is a natural number $m$ such that

(a) solutions $x, x^{\prime}$ in $C$ have $x \equiv x^{\prime} \bmod m$,

(b) there are $i \neq j$ such that either $\alpha_{i} \not \approx \alpha_{j}$ and $h\left(\alpha_{i} / \alpha_{j}\right) \geq \hbar$, or $\alpha_{i} \approx \alpha_{j}$ and $\operatorname{ord}\left(\alpha_{i}^{m} / \alpha_{j}^{m}\right) \leq \hbar^{-1}$.

Proof. We remark that $t \geq k$. Moreover, $t \geq n$. Indeed $\max t_{i}=n$, otherwise we had $M_{n}=0$, contradicting the hypothesis on the linear independece of $M_{1}, \ldots, M_{n}$ in the Proposition.

The case $k=1$ of the Proposition is trivial, as remarked at the beginning of [7], section 6. Assume $k \geq 2$ and $n=1$. In the proof, we replace lemma 8 of [6] by our lemma 2.4. Then, the last equation of [7], section 6, p. 625 can be replaced by

$$
\begin{aligned}
G(k)+2^{k} \cdot k^{3 k^{2}} & =(8 k)^{4(k-1)^{3} k^{2}}+2^{k} \cdot k^{3 k^{2}} \\
& \leq(8 k)^{4(k-1)^{3} k^{2}+3 k^{2}} \leq H(k)=H(T)
\end{aligned}
$$

because $4(x-1)^{3} x^{2}+3 x^{2} \leq 4(x-1) x^{4}$ for $x \geq 2$ and in addition $n=1$ yelds $T=k$.

As in [7] section 7, we assume $k \geq 2$ and $n \geq 2$. Thus

$$
T=\min \left(k^{n}, e^{12 t}\right) \geq \min \left(2^{n}, e^{12 n}\right)=2^{n} .
$$

Again, we replace lemma 8 of [6] by our lemma 2.4. Then, [7] equation (7.11) is replaced by (use $\left.(3 T)^{n} \leq(8 T)^{T}\right)$

$$
\left|\mathcal{S}^{\prime \prime}\right| G(T)<(3 T)^{n}(8 T)^{4(T-1)^{3} T^{2}}<(8 T)^{T+4(T-1)^{3} T^{2}} .
$$

The estimate at the end of [7], p. 630 becomes (use $3^{n} \leq 4^{n} \leq T^{2}$ )

$$
\begin{aligned}
& (8 T)^{T+4(T-1)^{3} T^{2}}+\exp \left(5 T^{3}+3^{n} T\right) \\
& \leq(8 T)^{T+4(T-1)^{3} T^{2}}+\exp \left(6 T^{3}\right) \\
& \leq(8 T)^{T+4(T-1)^{3} T^{2}+6 T^{3}} \\
& \leq H(T)
\end{aligned}
$$

because $x+4(x-1)^{3} x^{2}+6 x^{3} \leq 4(x-1) x^{4}$ for $x \geq 3$ and $T \geq 2^{n} \geq 4$. 


\section{Conclusion}

We closely follow [7], section 3 . For a non-zero polynomial $P \in \mathbb{C}[X]$ we recall that $t(P)=1+\operatorname{deg}(P)$ (with the convention $t(0)=0$ ). For a vector $\mathbf{P}=\left(P_{1}, \ldots, P_{k}\right) \in(\mathbb{C}[X])^{k}$, as before we put $t(\mathbf{P})=t\left(P_{1}\right)+\cdots+t\left(P_{k}\right)$ and $a(\mathbf{P})=\max _{i} t\left(P_{i}\right)$. Moreover, we let

$$
T(\mathbf{P})=\min \left(k^{a(\mathbf{P})}, e^{12 t(\mathbf{P})}\right) .
$$

For a set of integers $\mathcal{Z}$, let $\nu(\mathcal{Z})$ be the minimum $\nu$ such that $\mathcal{Z}$ can be expressed as the union of $\nu$ arithmetic progressions. We agree that single elements of $\mathbb{Z}$ are trivial arithmetic progressions and that $\nu(\mathcal{Z})=\infty$ if $\mathcal{Z}$ cannot be expressed as such a union. Notice that for a finite set $\mathcal{Z}, \nu(\mathcal{Z})$ is simply the cardinality of $\mathcal{Z}$.

Let $\left\{u_{m}\right\}$ be a linear recurrence of order $t$ with companion polynomial $\mathcal{P}$. Write

$$
\mathcal{P}(z)=c_{0} \prod_{i=1}^{k}\left(z-\alpha_{i}\right)^{a_{i}}
$$

with distinct roots $\alpha_{1}, \ldots, \alpha_{k}$. Then

$$
u_{m}=P_{1}(m) \alpha_{1}^{m}+\cdots+P_{k}(m) \alpha_{k}^{m}
$$

where $P_{i}$ is a polynomial of degree $<t_{i}(i=1, \ldots, k)$. Thus, we have to consider the polynomial-exponential equation

$$
P_{1}(x) \alpha_{1}^{x}+\cdots+P_{k}(x) \alpha_{k}^{x}=0 .
$$

Let $\mathcal{Z}=\mathcal{Z}(\mathbf{P})$ be the set of integer $x$ satisfying this equation. We have $t=t(\mathbf{P})$. Put for simplicity $a=a(\mathbf{P})=\max a_{i}$ and $T=T(\mathbf{P})$.

3.1. Proof of theorem 1.1. By induction on $t$, we prove that

$$
\nu(\mathcal{Z}) \leq Z(t, T)=(8 T)^{\left(2^{t}-1\right) 8 T^{5}} .
$$

As in [7], we may suppose $k \geq 2$ and $t \geq 3$. Since $k \geq 2$, we have $k^{a-1} \geq a$. Thus $k^{a} \geq k a \geq t$ and $T \geq t \geq 3$.

We consider the solutions of [7], equation (3.9)

$$
\sum_{r=1}^{n}\left(\sum_{j=1}^{a} c_{j, r} x^{j-1}\right) M_{r}\left(\alpha_{1}^{x}, \ldots, \alpha_{k}^{x}\right)=0 .
$$

There are fewer than $a$ numbers $x \in \mathbb{Z}$ such that each polynomial $\sum_{j=1}^{a} c_{j, r} x^{j-1}$ $(r=1, \ldots, n)$ vanishes. For other solutions of $(3.2)$ the numbers $M_{r}\left(\alpha_{1}^{x}, \ldots, \alpha_{k}^{x}\right)$ $(r=1, \ldots, n)$ are linearly independent over $\mathbb{Q}$. By the Proposition 2.5 , these numbers falls into at most

$$
H(T)=(8 T)^{4(T-1) T^{4}}
$$

classes. Fix one class $C$.

Proposition 2.5 leads to two cases. Let us consider first the case where there are $i \neq j$ such that $\alpha_{i} \approx \alpha_{j}$ and $\operatorname{ord}\left(\alpha_{i}^{m} / \alpha_{j}^{m}\right) \leq \hbar(T)^{-1}$. In this case, 
by [7], equation (3.12) and by the inequality just before (3.12), the set $\mathcal{Z}_{C}$ of solutions in our class satisfies

$$
\begin{aligned}
\nu\left(\mathcal{Z}_{C}\right) & \leq \exp \left(6 T^{4}\right) Z(t-1, T) \\
& \leq(8 T)^{4(T-1) T^{4}} Z(t-1, T)
\end{aligned}
$$

(use $6 x^{4} \leq 4(x-1)^{2} x^{4}$ for $x \geq 3$ ).

We now consider the case where there are $i \neq j$ such that $\alpha_{i} \not \approx \alpha_{j}$ and $h\left(\alpha_{i} / \alpha_{j}\right) \geq \hbar(T)$. We replace [6], lemma 7 by our lemma 2.3. Let, as in such lemma,

$$
F=(8 t)^{4(t-1)^{4}(t+2)}+5 E \log E .
$$

In the present situation, thanks to the inequality just before [7], equation (3.14), it hods $E \log E<\exp \left(8 T^{4}\right)$. Thus (3.14) is replaced by

$$
\begin{aligned}
F & <(8 t)^{4(t-1)^{4}(t+2)}+5 \exp \left(8 T^{4}\right) \\
& \leq 6(8 T)^{\max \left(4(T-1)^{4}(T+2), 4 T^{4}\right)} \\
& \leq(8 T)^{4(T-1) T^{4}}
\end{aligned}
$$

(recall that $t \leq T$ and use $e \leq \sqrt{8}, 1+\max \left(4(x-1)^{4}(x+2), 4 x^{4}\right) \leq 4(x-1) x^{4}$ for $x \geq 3$ ). Inequality (3.17) of [7] now reads

$$
\nu\left(\mathcal{Z}_{C}\right) \leq F Z(t-1, T)^{2}<(8 T)^{4(T-1) T^{4}} Z(t-1, T)^{2} .
$$

Therefore, in both cases of Proposition 2.5,

$$
\nu\left(\mathcal{Z}_{C}\right) \leq(8 T)^{4(T-1) T^{4}} Z(t-1, T)^{2} .
$$

Thus, using the new value (2.3) of $H(T)$ in the Proposition 2.5 and the inductive hypothesis, the inequality which follows (3.17) in [7] becomes

$$
\begin{aligned}
\nu(\mathcal{Z}) & <a+H(T)(8 T)^{4(T-1) T^{4}} Z(t-1, T)^{2} \\
& \leq T+(8 T)^{4(T-1) T^{4}+4(T-1) T^{4}+2\left(2^{t-1}-1\right) 8 T^{5}} \\
& \leq(8 T)^{1-8 T^{4}+\left(2^{t}-1\right) 8 T^{5}} \leq Z(t, T) .
\end{aligned}
$$

Hence (3.1) is established. Since $T \leq e^{12 t}$, we deduce

$$
\begin{aligned}
\nu(\mathcal{Z}) & \leq\left(8 e^{12 t}\right)^{2^{t} \cdot 8 e^{60 t}} \\
& \leq \exp \exp (t \log 2+\log 8+60 t+\log (\log 8+12 t)) \\
& \leq \exp \exp (70 t) .
\end{aligned}
$$

3.2. Proof of theorem 1.2. We follow the previous proof. We show by induction on $t$ that

$$
|\mathcal{Z}| \leq Z(t, T)=(8 T)^{8 T^{5} t}
$$

As in the proof of theorem 1.1, Proposition 2.5 leads to two cases. However, the case

$$
\exists i \neq j, \quad \alpha_{i} \approx \alpha_{j}, \quad \operatorname{ord}\left(\alpha_{i}^{m} / \alpha_{j}^{m}\right) \leq \hbar(T)^{-1}
$$

does not occur, since $\left\{u_{m}\right\}$ is not degenerate. More importantly, the case

$$
\exists i \neq j, \quad \alpha_{i} \not \approx \alpha_{j}, \quad h\left(\alpha_{i} / \alpha_{j}\right) \geq \hbar(T)
$$


has no additional troubles with non-trivial arithmetic progressions (see the paragraph in [7] between equations (3.14) and (3.15)). Thus, inequality (3.3) can be replaced by

$$
\nu\left(\mathcal{Z}_{C}\right) \leq F Z(t-1, T)<(8 T)^{4(T-1) T^{4}} Z(t-1, T)
$$

saving a square on $Z(t-1, T)$. In turns, (3.4) becomes

$$
\begin{aligned}
\nu(\mathcal{Z}) & <a+H(T)(8 T)^{4(T-1) T^{4}} Z(t-1, T) \\
& \leq T+(8 T)^{4(T-1) T^{4}+4(T-1) T^{4}+(t-1) 8 T^{5}} \\
& \leq(8 T)^{1-8 T^{4}+8 T^{5} t} \leq Z(t, T) .
\end{aligned}
$$

Hence (3.5) is established. Since $t \leq T \leq k^{n} \leq k^{a}$ and $k \geq 2$, we immediately deduce that

$$
\begin{aligned}
|\mathcal{Z}| \leq\left(8 k^{a}\right)^{8 k^{6 a}} & =\exp \left(8 k^{6 a}(3 \log 2+a \log k)\right) \\
& \leq \exp \left(32 a k^{6 a} \log k\right)
\end{aligned}
$$

As mentioned in the introduction, in theorem 1.1, we could try to combine our improvement with Allen's refined version of [7], lemma 2, to obtain a lower bound of the shape

$$
\exp \exp (c \sqrt{t} \log t) .
$$

We are not able to do that. In the degenerate case the growth in $t$ of $Z(t, T)$ is double exponential. So, as for theorem 1.2, we are not able to get further advantage. Neither in the non-degerate case we can obtain a bound of the kind (3.6). Allen takes

$$
H(T)=\exp \left(4(6 T)^{3 T}\right)
$$

in the main Proposition (se [2], Proposition in section 5.6) and replace

$$
T=\min \left(k^{n}, e^{12 t}\right)
$$

by $\min \left(k^{n}, e^{\sqrt{2} t}\right)$. This does not work with a function $H(T)$ which has a simple exponential growth. Indeed, the number of systems of 3-elements sets has a double exponential growth in $n$ ([6], section 11).

\section{REFERENCES}

[1] F. Amoroso and E. Viada. "Small points on subvarieties of a torus", to appear in Duke math. Journal. 150 vol. 3 (2009).

[2] P. Allen. "Multiplicity of linear recurrence sequences". M. Math thesis, University of Waterloo, Waterloo, Canada. 2006.

[3] P. Allen. "On the multiplicity of linear recurrence sequences". J. Number Theory, 126, no. 2, 212-216. (2007).

[4] J.-H. Evertse, H.-P. Schlickewei and W. Schmidt, "Linear equations in variables which lie in a finitely generated group", Annals of Math., 155, 807-836, (2002).

[5] W. M. Schmidt. "Heights of points on subvarieties of $\mathbb{G}_{\mathrm{m}}^{n}$ ". In "Number Theory 93-94", S. David editor, 157-187. London Math. Soc. Ser., volume 235, Cambridge University Press, 1996.

[6] W. M. Schmidt. "The zero multiplicity of linear recurrence sequences." Acta Math., 182, no. 2, 243-282 (1999). 
[7] W. M. Schmidt. "Zeros of linear recurrence sequences." Dedicated to Professor Kálmán Györy on the occasion of his 60th birthday. Publ. Math. Debrecen, 56, no. 3-4, 609-630 (2000). 